\title{
Análise da Doença Periodontal em pacientes Fumantes abrangendo os Efeitos Deletérios do Cigarro na perda de Inserção Clínica: Revisão de Literatura
}

\author{
Thamires Correia dos Santos Meneses ${ }^{1}$, Karina Sarno Paes Alves Dias ${ }^{2}$, \\ Rosivaldo de Araujo Campos Júnior ${ }^{3}$, Mariana Ilza Santos Simão ${ }^{4}$, Paulo Botelho Moura Junior ${ }^{5}$
}

\begin{abstract}
Resumo: A doença periodontal vem sendo cada vez mais frequente na população. Além da má higiene oral, o hábito de fumar constitui-se como um dos principais fatores de risco para essa patologia. Esse trabalho tem como objetivo realizar através de uma revisão da literatura, uma análise da doença periodontal em pacientes fumantes abrangendo os efeitos deletérios do cigarro na perda de inserção clínica. Foram selecionados artigos científicos publicados entre os anos de 2003 e 2018, através dos bancos de dados Pubmed, Scielo e Google Scholar. Nos estudos avaliados foi comprovado que o tabaco é capaz de aumentar a prevalência e progressão das doenças periodontais através das alterações que acometem os tecidos ósseo, conjuntivo e epitelial, a microbiota, o sistema imunológico e a microcirculação, ocasionando a perda dos tecidos de inserção.
\end{abstract}

Palavra-chave: Tabagismo. Saúde bucal. Periodontite.

\section{Periodontal Disease Analysis in Smoking Patients Covering the Deleterious Effects of Cigarette on Clinical Insertion Loss: A Literature Review}

\begin{abstract}
Periodontal disease has been increasingly common in the population. In addition to oral hygiene, tobacco use is considered as one of the main risk factors for this pathology. This paper aims to perform a literature review, an analysis of periodontal disease in patients that covers the deleterious effects of smoking on the loss of clinical insertion. We selected scientific articles published between 2003 and 2008 through the Pubmed, Scielo and Google Scholar databases. In the studies evaluated, ir has been shown that tobacco is able to increase the prevalence and progress of periodontal disease after changes in bone, connective and epithelial tissues, a microbiota, the immune system and microcirculation, leading to the loss of insertion tissues.
\end{abstract}

Keyword: Smoking. Oral health. Periodontitis

\footnotetext{
${ }^{1}$ Graduanda em Odontologia, Faculdade Independente do Nordeste - FAINOR, Vitória da Conquista, BA, Brasil. E-mail: Thamirescorreiaa@hotmail.com;

${ }^{2}$ Mestre em Periodontia/Especialista em implantodontia e Professora de Odontologia, Faculdade Independente do Nordeste FAINOR, Vitória da Conquista, BA, Brasil. E-mail: Karinasarnopad@gmail.com;

${ }^{3}$ Graduando em Odontologia, Faculdade Independente do Nordeste - FAINOR, Vitória da Conquista, BA, Brasil. E-mail: Rosivaldo-junior@hotmail.com;

${ }^{4}$ Graduanda em Odontologia, Faculdade Independente do Nordeste - FAINOR, Vitória da Conquista, BA, Brasil. E-mail: mari.simao.9@ hotmail.com;

${ }^{5}$ Graduando em Odontologia, Faculdade Independente do Nordeste - FAINOR, Vitória da Conquista, BA, Brasil.

E-mail: mourajr2015@ @otmail.com.
} 


\section{Introdução}

A doença periodontal é uma das condições de saúde bucal mais importantes que afetam as populações em todo o mundo (LORENZO, S.M. et al., 2015). Trata-se de uma patologia infecto-inflamatória, multifatorial, com caráter polimicrobiano e sua progressão ocorre pela presença do biofilme bacteriano, localizado supra e subgengival (CARVALHO; ROTBLAND; NOGUEIRA, 2017). Fatores microbiológicos, comportamentais, ambientais, alimentares, sistêmicos/metabólicos, imunológicos, anatômicos e genéticos contribuem para a suscetibilidade ao seu desenvolvimento e expressão clínica da doença (ALBUQUERQUE, et al., 2015).

Dependendo de sua gravidade, a doença periodontal pode ser classificada como gengivite ou periodontite. A gengivite tem sua resposta inflamatória limitada ao periodonto de proteção, enquanto a periodontite invade o periodonto de sustentação, causando perda do ligamento periodontal e destruição dos tecidos ósseos adjacentes (SANTOS; SIQUEIRA, 2016).

O hábito de fumar é considerado um fator de risco para as doenças periodontais e um dos problemas mais importantes de saúde pública. O fumo modifica a resposta imune do indivíduo contra os microrganismos periodontopatogênicos, comprometendo o sistema de defesa, o que pode gerar a perda de inserção periodontal e reabsorção óssea alveolar (LONDERO, A.C.C. et al., 2018).

Evidências clínicas mostram que fumantes possuem maior perda óssea, maior número de bolsas profundas e são mais susceptíveis ao desenvolvimento de lesões de bifurcação (ANTONINI, R. et al., 2013). Entretanto, podem apresentar menor inflamação gengival do que não fumantes (MARTELLI, J. et al, 2003).

Produtos químicos e toxinas da fumaça do cigarro podem retardar a cicatrização por inibir funções celulares básicas (PIASSI E., 2005). A revascularização do osso e tecidos moles é prejudicada pelo tabagismo, uma vez que, os componentes voláteis dos cigarros denominados acroleína e acetaldeído inibem a fixação e proliferação de fibroblastos gengivais (JACOB; VELLAPPALLY; SMEJIKALOVÁ, 2007). Os fibroblastos expostos à nicotina produzem menos fibronectina e colágeno e mais colagenase gerando efeitos negativos nas funções e 
influenciando na cicatrização de feridas assim como na progressão da periodontite (JACOB; VELLAPPALLY; SMEJIKALOVÁ, 2007).

Considerando a importância do processo educativo tanto para prevenção quanto para o sucesso do tratamento, o presente estudo tem por objetivo, através de uma revisão de literatura, fazer uma análise da doença periodontal nos pacientes fumantes, relatando os seus efeitos na perda de inserção clínica.

\section{Metodologia}

A presente revisão de literatura foi baseada em uma pesquisa bibliográfica em três das principais bases de dados mundiais, Pubmed, Scielo e Google Scholar, utilizando os descritores: doença periodontal, tabaco, periodontia, periodontite e gengivite, publicados entre 2003 e 2018. Os artigos encontrados na busca foram submetidos à análise, sendo avaliados pelo seu título e resumo. Foram excluídas teses, monografias, livros e capítulos. Ao final, restaram 29 artigos, abaixo discriminados na tabela 1 .

Tabela 1 - Artigos que fizeram parte do presente estudo.

\begin{tabular}{|c|c|c|}
\hline Autor, Título & Periódico & Ano \\
\hline $\begin{array}{l}\text { ALBUQUERQUE, E.C.M. et al. Prevalência da doença cárie e } \\
\text { periodontal em adolescentes de uma escola da rede particular de } \\
\text { Quixadá-CE. }\end{array}$ & Rev. Expressão católica, v.4, n.2, p.1-12. & 2015 \\
\hline $\begin{array}{l}\text { ACCARINI, R., GODOY, M.F. Doença periodontal como potencial } \\
\text { fator de risco para síndromes coronarianas agudas. }\end{array}$ & Arq Bras Cardiol, v.87, n.5, p.6-592. & 2006 \\
\hline ANTONINI, R. et al. Fisiopatologia da doença periodontal. & $\begin{array}{l}\text { Rev. Inova sáude, Criciúma, v.2, n.2, p.90- } \\
107 .\end{array}$ & 2013 \\
\hline $\begin{array}{l}\text { BAGAITKAR, J. et al. Tobacco Smoke Augments Porphyromonas } \\
\text { gingivalis - Streptococcus gordonii Biofilm Formation. }\end{array}$ & Plos one, v.6, n.11, p.1-8. & 2011 \\
\hline $\begin{array}{l}\text { BERNARDES, V.S., FERRES, M.O., LOPES JUNIOR, M. O } \\
\text { tabagismo e as doenças periodontais. }\end{array}$ & $\begin{array}{l}\text { Rev. Fol- Faculdade de odontologia de } \\
\text { lins.v.23, n.1, p.37-45. }\end{array}$ & 2013 \\
\hline $\begin{array}{l}\text { CAMARGO, G.A.C.G et al. Aspectos clínicos, microbiológicos e } \\
\text { tratamento periodontal em pacientes fumantes portadores de doença } \\
\text { periodontal crônica: revisão de literatura. }\end{array}$ & Rev. bras. odontol, v.73, n.4, p.325-30. & 2016 \\
\hline $\begin{array}{l}\text { CARVALHO, P.A.; ROTBLAND, M.; NOGUEIRA, A.C.O. A doença } \\
\text { periodontal como fator de risco para a pneumonia nosocominal. }\end{array}$ & $\begin{array}{l}\text { Rev. Fluminense de odontologia, v.52, n.2, } \\
\text { p.80-92. }\end{array}$ & 2017 \\
\hline $\begin{array}{l}\text { CATON, G.J. et al. A new classification scheme for periodontal and } \\
\text { peri-implant diseases and conditions - Introduction and key changes } \\
\text { from the } 1999 \text { classification. }\end{array}$ & J Clin Periodontol, 45(Suppl 20):S1-8. & 2018 \\
\hline $\begin{array}{l}\text { CRUZ, G.A. et al. Estudo clínico e radiográfico do nível da crista óssea } \\
\text { alveolar em pacientes fumantes. }\end{array}$ & Cienc Odontol Bras, v.6, n.4, p.60-9. & 2003 \\
\hline $\begin{array}{l}\text { EICK, S.; PIETKIEWICZ, M.; SCULEAN, A. Oral microbiota in Swiss } \\
\text { adolescentes. }\end{array}$ & Clin Oral Invest. & 2012 \\
\hline
\end{tabular}
31 Id on Line Rev. Mult. Psic. V.13, N. 48 p. 29-40, Dezembro/2019 - ISSN 1981-1179
Edição eletrônica em http://idonline.emnuvens.com.br/id 
FERREIRA, A.L., NUNES, L.H.A.C., MACIEL, A.A.B. Relação do tabagismo com a doença periodontal crônica.

IDE, R. et al. Periodontal disease and incident diabetes: a seven-year study.

HANIOKA, T. et al. Causal assessment of smoking and tooth loss: a systematic review of observational studies.

JACOB, V., VELLAPPALLY, S., SMEIJKALOVÁ J. The influence of cigarette smoking on various aspects of periodontal health.

KUBOTA, M. et al. Effect of smoking on subgingival microflora of patients with periodontitis in Japan.

LONDERO, A.C.C. et al. Nível de conhecimento dos fumantes do centro hiperdia- Juiz de fora, sobre doença periodontal.

LORENZO, S.M. et al. Periodontal conditions and associated factors among adults and the elderly: findings from the first National Oral Health Survey in Uruguay.

MARTELLI, J. et al. Comparação clínica da presença de lesões de furca entre pacientes fumantes e não-fumantes.

MATOS, G.R.M.; GODOY, M.F. Influência do tabagismo no tratamento e prognóstico da doença periodontal.

MEDEIROS, G.V.P.; DIAS, K.S.P.A. A influência do tabagismo na doença periodontal: Uma revisão de literatura.

MONTEIRO-DA-SILVA, F. et al. Characterization of the oral fungal microbiota in smokers and non-smokers.

MUZOROVIC, S. et al. The Relationship Between Oral Hygiene and Oral Colonization with Candida Species.

PIASSI, E. O fumo como fator modificador da doença periodontal.

RIBEIRO, I.L.A., VELOSO, H.H.P. Influência do tabagismo nas alterações pulpares

SANTOS, V.T.A.; SIQUEIRA, L.C.B. Tabaco e doenças periodontais.

SEIXAS, A.R. et al. Prevenção e tratamento da gengivite na pratica do técnico em saúde bucal.

SOUTO, G.R. et al. Avaliação do infiltrado inflamatório na gengivite crônica de indivíduos fumantes e não fumantes.

STEFFENS, J.P.; MARCANTONIO, R.A.C. Classificação das doenças e condições periodontais e pre-implantares 2018: guia Praticos e Ponto chave.

VINHAS, A.S., PACHECO, J.J. Tabaco e doenças periodontais.
Rev. Invest. Biomédica, v.1, n.1, p.50-59.

Dent Res, v.90, n.1, p.6-41.

BMC Public Health, v.11, n.221, p. 2-10.

Acta Medica, v.50, n.1, p.3-5.

BMC Oral Health, v.11, n.1, p.6-11.

Braz J Periodontol, v.18, n.1, p.7-11.

Cad. Saúde Pública, v.31, n.11, p.65-74 2015

Rev Bras Cir Period, v.1, n.1, p.9-26,.

Arq Cienc Saúde, v.18, n.1, p.8-55.

Id on Line, Rev. Multidisciplinar e de Psicologia, v.12, n.40, p.470-479.

Eur J Oral Sci, v.121, n.2, p.5-132.

Med Arh, v.66, n.6, p.7-415

Rev Int Periodontia Clin, v.2, n.5, p.67-73.

Rev. Odontol Bras Central,v.21, n.58, p.570-575.

2012

Rev. Cient. In FOC, v.1, n.1, p.13-24.

Rev. Gestão \& Saúde. v.1, n.2, p.37-41.

2016

2010

Braz J Periodontol. v.23, n.2, p.11-15.

Rev Odontol UNESP, v.47, n.4, p.189-197. n.1, p.49-39. s 3 $=$

Fonte: Dados da pesquisa.

A distribuição anual dos artigos demonstrou maior concentração nos anos de 2011 (5 artigos), 2018 (4 artigos) e 2012 (3 artigos). Na sequência o gráfico 1 apresenta a distribuição por classe quadrianual da quantidade dos artigos usados neste estudo. 
Gráfico 1 - Distribuição da quantidade dos artigos usados neste estudo, por classe quadrianual

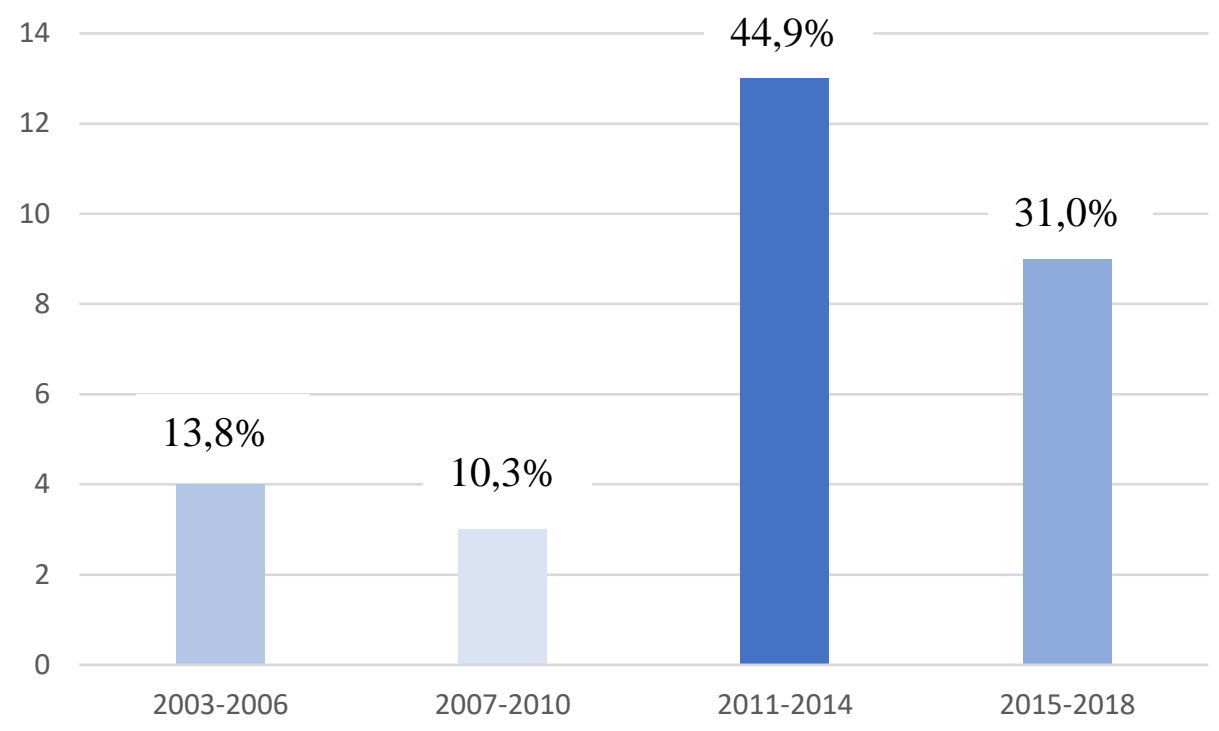

Fonte: Dados da pesquisa.

Observou-se uma maior concentração de artigos sobre a temática entre 2011 a 2014 (44,9\%). O interesse parece manter-se ainda entre 2015 a 2018 (31,0\%), embora em menor intensidade.

\section{Revisão da Literatura}

\section{Doença periodontal}

A doença periodontal ocorre pela colonização de bactérias gram-negativas e anaeróbias, como Porphyromonas gingivalis e Actinobacillus actinomycetemcomitans (ARCCARINI; GODOY, 2006). Evolui continuamente com períodos de exacerbação e de remissão, resultando em resposta inflamatória e imune do hospedeiro à presença do biofilme dental (CARMAGO G.A.C.G. et al., 2016). 
Segundo a American Academy of Periodontology (AAP, 2018), a doença periodontal é classificada em: Saúde gengival, Gengivite, Periodontite leve, Periodontite moderada e Periodontite severa.

A gengivite é definida como uma inflamação decorrente da presença de bactérias localizadas na margem gengival, podendo expandir por toda unidade gengival remanescente (ANTONINI, R. et al., 2013). Suas características iniciais se baseiam em alteração da cor da gengiva para um tom avermelhado, aumento de volume, presença de sangramento e alterações na consistência, podendo ou não apresentar mau hálito (SEIXAS, A.R. et al., 2010).

Quando as modificações patológicas observadas na gengivite aumentam até a destruição do ligamento periodontal e tecidos adjacentes, passa a ser chamada de periodontite (SANTOS; SIQUEIRA, 2016) e sua classificação por estágios relacionada de acordo com a severidade da doença (STEFFENS; MARCANTONIO, 2018).

\section{Fatores de risco da doença periodontal}

A participação das bactérias na doença periodontal está envolvida na etiologia das diferentes formas de doenças periodontais, essencialmente nas severas (CARVALHO; ROTBLAND; NOGUEIRA, 2017). A deficiência na higienização bucal promove a formação de uma película pegajosa de bactérias que se aderem ao dente, caracterizando-se como biofilme dental (SEIXAS, A.R. et al., 2010).

De acordo com a Classificação das Doenças Periodontais, 2018, (CATON, G. J. et al., 2018), os fatores de risco predisponentes se dividem em locais e sistêmicos. Os fatores de risco locais incluem aqueles que promovem a retenção do biofilme dental como margens de restaurações desadaptadas e secura bucal. Já como riscos sistêmicos estão o tabagismo, hiperglicemia, fatores nutricionais, agentes farmacológicos, hormônios esteroides sexuais e condições hematológicas.

A doença periodontal encontra-se associada a baixas condições socioeconômicas, dificuldade de acesso aos serviços de saúde, assim como o tabagismo, alcoolismo, dieta rica em carboidratos, hipertensão arterial sistêmica, diabetes, obesidade, síndrome metabólica, estresse oxidativo, osteoporose na pós-menopausa e higiene bucal deficiente (IDE, R. et al., 2011). 


\section{Doença periodontal e tabaco}

A associação da doença periodontal com o tabaco é um fator de risco significativo independente para o início, extensão e gravidade da doença periodontal. $\mathrm{O}$ individuo que possui o hábito de fumar, apresenta maior recessão gengival e menor ganho de inserção, como também piores efeitos nos tratamentos periodontais (MATOS; GODOY, 2011).

Estudos mostram que o tabagista possui diversas alterações bucais como: diminuição da capacidade de defesa frente à placa bacteriana, variações no processo inflamatório e vascular degenerativo, comprometimento dos processos odontológicos dentinários e pulpares, influência negativa aos fibroblastos e à doença periodontal, aumentando sua severidade, incidência e dificuldade de tratamento (RIBEIRO; VELOSO, 2012).

Dentre as diversas substâncias tóxicas que se apresentam no tabaco, a nicotina e o monóxido de carbono são considerados os principais causadores de modificações imunológicas, diminuição da imunoglobulina $\mathrm{G}$ e danos nas funções das células de defesa. Além disso, apresentam efeito vasoconstrictor e atenuação do fluxo sanguíneo de forma crônica, ocasionando efeitos citotóxicos sobre tecidos e células, principalmente fibroblastos, modificando a microbiota patogênica e aumentando sua prevalência (FERREIRA; NUNES; MACIEL, 2014).

\section{Tecido ósseo}

A nicotina ao ser consumida pode diminuir a proliferação dos osteoblastos, aumentando a atividade da fosfatase alcalina. Também limita a síntese de colágeno, intervindo na deposição de proteína, impossibilitando a formação óssea, predispondo a doença periodontal (BERNARDES; FERRES; LOPES JUNIOR, 2013).

As áreas lesionadas sofrem com a falta de nutrição e o tecido de reparação mostra-se com elevada atividade metabólica, havendo a necessidade de suprimento sanguíneo e oxigênio. No entanto, sabendo-se que a nicotina favorece a diminuição dos mesmos, a reparação periodontal torna-se comprometida (VINHAS; PACHECO, 2008). 


\section{Tecido epitelial}

Em fumantes ocorre uma produção exacerbada de moléculas inflamatórias, ocasionando a destruição do tecido conjuntivo e do osso alveolar. Compostos que apresentam no tabaco, tais como: nicotina, acroleína e acetaldeído inibem função e proliferação de fibroblastos, produção de colágeno, adesão nas raízes e indução de citotoxicidade (BERNARDES; FERRES; LOPES JUNIOR, 2013).

\section{Microbiota}

Há influências entre hospedeiro e bactérias, originando-se em destruições mais agressivas, isso ocorre pela dificuldade no equilíbrio dos mesmos, motivada na formação da placa subgengival, alterando a quantidade de virulência de patógenos e também na resposta do organismo (BERNARDES; FERRES; LOPES JUNIOR, 2013).

O fumo exerce influência na presença de microrganismos na placa, favorecendo a colonização e crescimento de espécies como as do complexo vermelho, Porphyromonas gingivalis e Tanerella forsythia e do complexo laranja, Prevotella intermedia, Prevotella nigrescens, Fusobacterium nucleatum, Campylobacter rectus e Parvimonas micra, associadas à doença periodontal (KUBOTA, M. et al., 2011).

Segundo Kubota et al. (2011), os fumantes são mais suscetíveis à infecção pela Porphyromonas gingivalis, já que se adaptam ao stress ambiental apresentado pelo cigarro, alterando a expressão de vários genes e proteínas da membrana externa que são essenciais em diminuir o seu potencial inflamatório.

Bagaitkar et al. (2011), expuseram a bactéria Porphyromonas gingivalis ao extrato de cigarro e observaram aumento do antígeno fimbrial A e diminuição da capacidade próinflamatória induzida pela bactéria, o que colaborou para um aumento da capacidade de produção de biofilme de Porphyromonas gingivalis, e potencializou sua interação com Streptococcus gordonii, confirmando a suspeita de que o tabagismo potencializa a formação de complexos entre bactérias da microbiota e bactérias anaeróbicas patogênicas envolvidas na periodontite.

36 Id on Line Rev. Mult. Psic. V.13, N. 48 p. 29-40, Dezembro/2019 - ISSN 1981-1179 
O tabagismo não só pode facilitar a colonização da cavidade oral por bactérias patogênicas, como também por bolores e leveduras patogênicas (MONTEIRO-DA-SILVA, F. et al., 2013). Muzurovic et al. (2012), avaliaram 77 pacientes fumantes (55\%) e 65 não fumantes (45\%) e encontraram Candida albicans em 40 pacientes (29\%), destes, $33(82.5 \%)$ eram fumantes, o que mostrou a existência de correlação entre o tabagismo e presença de Candida albicans na cavidade oral.

\section{Discussão}

A doença periodontal é proveniente de uma reação infecciosa, pela aglomeração de bactérias, resultando em uma inflamação que acomete os tecidos periodontais de suporte (SANTOS; SIQUEIRA, 2016). Sua manifestação e progressão são influenciadas por uma grande variedade de fatores de risco, como características do indivíduo, fatores sociais, comportamentais, sistêmicos e genéticos, e a composição microbiana do biofilme dental (EICK; PIETKIEWICZ; SCULEAN, 2012). No entanto, Siqueira e Santos (2016) e Ribeiro e Veloso (2012) descrevem que o fumo é apontado como o principal fator determinante à doença periodontal.

Para Vinhas e Pacheco (2008), os efeitos tóxicos do tabaco, têm consequências mais pela absorção sistêmica do que pela tópica na cavidade oral, sendo nocivos ao periodonto após consumo crônico e prolongado.

A literatura confirma que há relação direta entre o uso do fumo e o aumento da perda de inserção e profundidade de bolsa, assim como diminuição da crista óssea. De acordo com a frequência de consumo de cigarros e da idade, o estudo do nível da crista óssea alveolar na região mesial e distal de pré-molares maxila e mandíbula de pacientes não fumantes, exfumantes e fumantes revelou que ocorre aumento da distância crista óssea alveolar à junção cemento esmalte para os fumantes, quando comparado aos ex-fumantes e não fumantes, sendo que essa perda óssea independe da presença de doença periodontal (CRUZ, G.A. et al., 2003).

Os pacientes fumantes têm também maior predomínio e gravidade de envolvimento de furca, avaliada por evidência clínica e radiográfica de perda óssea (BERNARDES; FERRES; LOPES JUNIOR, 2013). 
É importante salientar que, normalmente, os fumantes apresentam menor inflamação clínica e sangramento gengival do que os não fumantes portadores da doença periodontal. Isso pode ser atribuído ao efeito vasoconstritor da nicotina, que reduz o fluxo sanguíneo, edema e sinais clínicos da inflamação, mascarando doenças periodontais graves em fumantes HANIOKA, T. et al., 2011). Além disso, a vasoconstrição promove diminuição da angiogênese, dos níveis de adesão, proliferação e diferenciação das células ósseas (RIBEIRO; VELOSO, 2012).

Segundo Bernardes, Ferres e Lopes Junior (2013), o efeito térmico da fumaça também produz efeitos maléficos, aumentando a perda de inserção e retração nas superfícies linguais dos dentes superiores e incisivos inferiores.

O fumo afeta a função dos linfócitos B e T, reduzem as classes das imunoglobulinas e inibe citocinas pró-inflamatórias (SOUTO, G.R. et al., 2013). Ribeiro e Veloso (2012) acrescentam ainda a liberação diminuída de IL1- $\beta$ (Interleucina 1-beta) e TNF- $\alpha$ (Fator de Necrose Tumoral alfa).

O hábito de fumar também pode reduzir a absorção intestinal do cálcio, dificultando a função dos osteoblastos e induzindo a perda óssea (BERNARDES; FERRES; LOPES JUNIOR, 2013).

A literatura mostra que o tratamento periodontal é prejudicado pelo fumo com aumento do risco de fracasso devido a interferência negativa no processo de cicatrização. Felizmente, alguns autores enfatizam que a cessação do fumo pode diminuir os danos causados (KUBOTA et al., 2011).

\section{Conclusão}

Com base nas evidências encontradas nesse estudo conclui-se que o tabaco é capaz de aumentar a prevalência e progressão das doenças periodontais através das alterações que acometem os tecidos ósseo, conjuntivo e epitelial, a microbiota, o sistema imunológico e a microcirculação, ocasionando a perda dos tecidos de inserção. 


\section{Referências}

ALBUQUERQUE, E.C.M. et al. Prevalência da doença cárie e periodontal em adolescentes de uma escola da rede particular de Quixadá-CE. Rev. Expressão católica, v.4, n.2, p.1-12, 2015.

ACCARINI, R., GODOY, M.F. Doença periodontal como potencial fator de risco para síndromes coronarianas agudas. Arq Bras Cardiol, v.87, n.5, p.6-592, 2006.

ANTONINI, R. et al. Fisiopatologia da doença periodontal. Rev. Inova sáude, Criciúma, v.2, n.2, p.90$107,2013$.

BAGAITKAR, J. et al. Tobacco Smoke Augments Porphyromonas gingivalis - Streptococcus gordonii Biofilm Formation. Plos one, v.6, n.11, p.1-8, 2011.

BERNARDES, V.S., FERRES, M.O., LOPES JUNIOR, M. O tabagismo e as doenças periodontais. Rev. Fol- Faculdade de odontologia de lins.v.23, n.1, p.37-45, 2013.

CAMARGO, G.A.C.G et al. Aspectos clínicos, microbiológicos e tratamento periodontal em pacientes fumantes portadores de doença periodontal crônica: revisão de literatura. Rev. bras. odontol, v.73, n.4, p.325-30, 2016.

CARVALHO, P.A.; ROTBLAND, M.; NOGUEIRA, A.C.O. A doença periodontal como fator de risco para a pneumonia nosocominal. Rev. Fluminense de odontologia, v.52, n.2, p.80-92, 2017.

CATON, G.J. et al. A new classification scheme for periodontal and peri-implant diseases and conditions - Introduction and key changes from the 1999 classification. J Clin Periodontol, 2018;45(Suppl 20):S1-8. http:// dx.doi.org/10.1111/jcpe.12935. PMid:29926489.

CRUZ, G.A. et al. Estudo clínico e radiográfico do nível da crista óssea alveolar em pacientes fumantes. Cienc Odontol Bras, v.6, n.4, p.60-9, 2003.

EICK, S.; PIETKIEWICZ, M.; SCULEAN, A. Oral microbiota in Swiss adolescentes. Clin Oral Invest. 2012.

FERREIRA, A.L., NUNES, L.H.A.C., MACIEL, A.A.B. Relação do tabagismo com a doença periodontal crônica. Rev. Invest. Biomédica, v.1, n.1, p.50-59, 2014.

IDE, R. et al. Periodontal disease and incident diabetes: a seven-year study. Dent Res, v.90, n.1, p.6-41, 2011.

HANIOKA, T. et al. Causal assessment of smoking and tooth loss: a systematic review of observational studies. BMC Public Health, v.11, n.221, p. 2-10, 2011.

JACOB, V., VELLAPPALLY, S., SMEIJKALOVÁ J. The influence of cigarette smoking on various aspects of periodontal health. Acta Medica, v.50, n.1, p.3-5, 2007.

KUBOTA, M. et al. Effect of smoking on subgingival microflora of patients with periodontitis in Japan. BMC Oral Health, v.11, n.1, p.6-11, 2011). 
LONDERO, A.C.C. et al. Nível de conhecimento dos fumantes do centro hiperdia- Juiz de fora, sobre doença periodontal. Braz J Periodontol, v.18, n.1, p.7-11, 2018.

LORENZO, S.M. et al. Periodontal conditions and associated factors among adults and the elderly: findings from the first National Oral Health Survey in Uruguay. Cad. Saúde Pública, v.31, n.11, p.6574,2015

MARTELLI, J. et al. Comparação clínica da presença de lesões de furca entre pacientes fumantes e nãofumantes. Rev Bras Cir Period, v.1, n.1, p.9-26, 2003.

MATOS, G.R.M.; GODOY, M.F. Influência do tabagismo no tratamento e prognóstico da doença periodontal. Arq Cienc Saúde, v.18, n.1, p.8-55, 2011.

MEDEIROS, G.V.P.; DIAS, K.S.P.A. A influência do tabagismo na doença periodontal: Uma revisão de literatura. Id on Line, Rev. Multidisciplinar e de Psicologia, v.12, n.40, p.470-479, 2018.

MONTEIRO-DA-SILVA, F. et al. Characterization of the oral fungal microbiota in smokers and nonsmokers. Eur J Oral Sci, v.121, n.2, p.5-132, 2013).

MUZOROVIC, S. et al. The Relationship Between Oral Hygiene and Oral Colonization with Candida Species. Med Arh, v.66, n.6, p.7-415, 2012).

PIASSI, E. O fumo como fator modificador da doença periodontal. Rev Int Periodontia Clin, v.2, n.5, p.67-73, 2005.

RIBEIRO, I.L.A., VELOSO, H.H.P. Influência do tabagismo nas alterações pulpares. Rev. Odontol Bras Central,v.21, n.58, p.570-575, 2012.

SANTOS, V.T.A.; SIQUEIRA, L.C.B. Tabaco e doenças periodontais. Rev. Cient. In FOC, v.1, n.1, p.13-24, 2016.

SEIXAS, A.R. et al. Prevenção e tratamento da gengivite na pratica do técnico em saúde bucal. Rev. Gestão \& Saúde. v.1, n.2, p.37-41, 2010.

SOUTO, G.R. et al. Avaliação do infiltrado inflamatório na gengivite crônica de indivíduos fumantes e não fumantes. Braz J Periodontol. v.23, n.2, p.11-15, 2013.

STEFFENS, J.P.; MARCANTONIO, R.A.C. Classificação das doenças e condições periodontais e preimplantares 2018: guia Praticos e Ponto chave. Ver Odontol UNESP, v.47, n.4, p.189-197, 2018.

VINHAS, A.S., PACHECO, J.J. Tabaco e doenças periodontais. Rev. Port Estomatol Cir Maxilofac, v.49, n.1, p.49-39, 2008.

\section{Como citar este artigo (Formato ABNT):}

MENESES, Thamires Correia dos Santos; DIAS, Karina Sarno Paes Alves; CAMPOS JÚNIOR, Rosivaldo de Araujo; SIMÃO, Mariana Ilza Santos; MOURA JUNIOR, Paulo Botelho; Análise da Doença Periodontal em pacientes Fumantes abrangendo os Efeitos Deletérios do Cigarro na perda de Inserção Clínica: Revisão de Literatura. Id on Line Rev.Mult. Psic., Dezembro/2019, vol.13, n.48, p. 29-40. ISSN: 1981-1179.

Recebido: 29/10/2019; Aceito: 04/11/2019. 